\title{
Small-pad Resistive Micromegas for Operation at Very High Rates
}

\section{P. lengo*}

CERN; E-mail: paolo.iengo@cern.ch

\section{Alviggi, M.T. Camerlingo, V. Canale, M. Della Pietra, C. Di Donato, C. Grieco}

University of Naples and INFN, Naples

\section{Biglietti, M. lodice}

INFN Roma Tre, Rome

\section{F. Petrucci, E. Rossi, V. Vecchio}

University Roma Tre and INFN Roma Tre, Rome

\section{E. Farina}

University of Pavia and CERN

\section{S. Franchino}

University of Wurzburg

\section{G. Sekhniaidze}

\author{
INFN Naples
}

\section{O. Sidiropoulou}

University of Wurzburg and CERN

\begin{abstract}
Resistive Micromegas detectors have proved to be suitable for precision tracking in dense particle rate environment up to few $\mathrm{kHz} / \mathrm{cm}^{2}$. Rate capability up to few $\mathrm{MHz} / \mathrm{cm}^{2}$ and low occupancy can be achieved by using few $\mathrm{mm}^{2}$ readout pads. Such a rate capability will be required in upgrades of forward muon detectors of LHC experiments as well as in experiments at future colliders. We present the development of resistive micromegas with $\mathrm{O}\left(\mathrm{mm}^{2}\right)$ pad readout aiming at precision tracking in high rate environment without efficiency loss up to several $\mathrm{MHz} / \mathrm{cm}^{2}$.

A first small-pad prototype has been designed, constructed and tested. It consists of a matrix of $48 \times 16$ pads. Each pad has rectangular shape with a pitch of 1 and $3 \mathrm{~mm}$ in the two coordinates. The active surface is $4.8 \times 4.8 \mathrm{~cm}^{2}$ with a total number of 768 channels. Characterization and performance studies of the detector have been carried out by means of radioactive sources, high irradiation X-Rays and test beam. The results will be presented, along with recent developments aiming at the construction of fully scalable, thousands-channels small-pad detectors.
\end{abstract}

The European Physical Society Conference on High Energy Physics

5-12 July, 2017

Venice

${ }^{*}$ Speaker. 


\section{Introduction}

Resistive Micromegas [1] demonstrated to be a solid detector technology for HEP experiments. Developed for the upgrade of the ATLAS Muon [2] system, they exploit resistive anode strips to suppress discharges. Resistive Micromegas 'a la ATLAS provide: high efficiency and good tracking capability $\left(\sim 100 \mu \mathrm{m}\right.$ for tracks with inclination up to $\left.30^{\circ}\right)$; operation at a rate up to $<100 \mathrm{kHz} / \mathrm{cm}^{2}$; possibility to be industrially produced.

Several features need to be optimized in order to reach higher rate capabilities. These include: faster charge evacuation on resistive electrode; reduction of the detector Pad Response Function; reduction of the occupancy. Figure 1 shows the pad size as function of the maximum tolerable rate for a signal pulse width ranging between 50 and $500 \mathrm{~ns}$. Assuming a pusle widht in the interval [100-200]ns, rate capabilities of few $\mathrm{MHz} / \mathrm{cm}^{2}$ can be only reached by designing a detector with a pad size of few $\mathrm{mm}^{2}$.

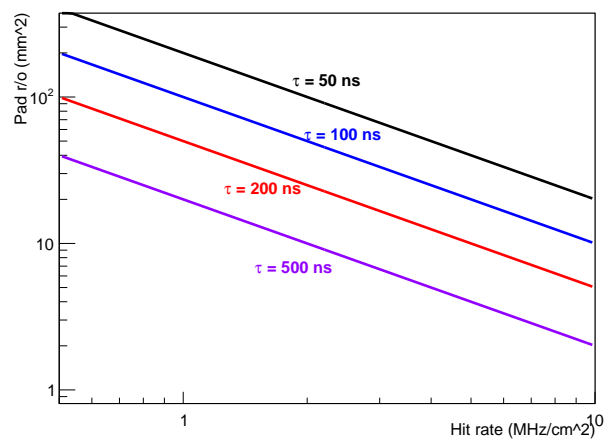

Figure 1: Required maximum pad readout size as function of the maximum particle rate to limit the efficiency drop to $10 \%$ for a generic gaseous detector. The four curves correspond to different signal pulse width, which includes the contribution of the front end electronics [3].

Here we present the development and first test results of Micromegas with small resistive pads for high rate application. The final goal is to develp a detector able to efficiently work at particle rates up to several $\mathrm{MHz} / \mathrm{cm}^{2}$, providing $2 \mathrm{D}$ tracking capability.

\section{Detector layout}

Figure 2 left shows the schematic concept of the first small-pad Micromegas prototype detector built at CERN in 2015 [4]. The readout plane is segmented in pads $0.8 \times 2.8 \mathrm{~mm}^{2}$ wide with a pitch of $1 \times 3 \mathrm{~mm}^{2}$, covering a surface of $4.8 \times 4.8 \mathrm{~mm}^{2}$ for a total number of 768 channels. The resistive protection is obtained with a double layer of embedded resistors separated by a $12.5 \mu \mathrm{m}$ thick Kapton foil. The resistors have the same size as the readout pads. The connection between the two layers of resistors and the readout pads is done with micro-vias in the Kapton, filled with silver-epoxy paste deposited by screen-printing. The vias on the two layer are staggered such that the electron current evaquated throught the reaodut pads always sees at least one full embedded resistor. The resistance between a pad on the uppermost resistive layer and the corresponding 
readout pad ranges between 3 and $7 \mathrm{M} \Omega$. Figure 2 right shows the top layer of the small-pad Micromegas detector board.

The detector is completed with a bulk-Micromegas [5] process, defining the $128 \mu \mathrm{m}$ amplification gap with a metallic micro-mesh supported by Pyralux insulating pillars, and with a drift cathode defining the $5 \mathrm{~mm}$ wide conversion gap.

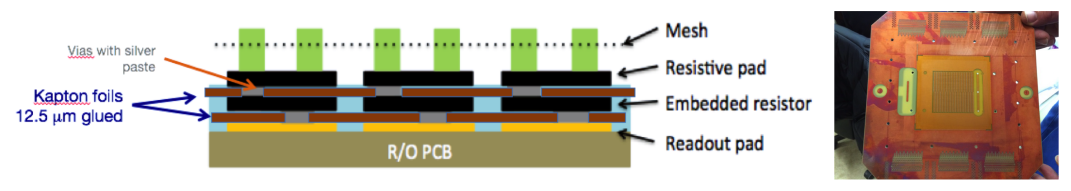

Figure 2: Left: Schematic of the small-pad Micromegas structure. Right: Picture of the detector board.

\section{Test results.}

A number of tests were performed in order to study the detector performance and the responce to different particle radiations. In the following the main results from tests done with ${ }^{55} \mathrm{Fe}$ source, $\mathrm{X}$-rays from $\mathrm{Cu}$ and particle beams are described. The first two tests were conducted at the Gas Detector Development (GDD) laboaratory at CERN, while the beam-test was run at the SPS H4 CERN experimantal area. The same gas mixture ( $\mathrm{Ar}: \mathrm{CO}_{2}$ 93:7) was used for all tests.

\subsection{Test with ${ }^{55} \mathrm{Fe}$ source.}

The detector was exposed to an ${ }^{55} \mathrm{Fe}$ source, the current read from the readout pads (at ground) and the trigger rate measured from the mesh. Figure 3 left shows the energy spectra aquired with a multi-channel analyzer for amplification voltages, applied to the mesh, ranging between 500 and $540 \mathrm{~V}$. The drift voltage was $\mathrm{V}_{\text {drift }}=200 \mathrm{~V}$. The energy resolution was measured to be of the order of $35 \%$. Figure 3 right shows the evolution of the current as function of the time: a charging-up effect creating a gain reduction of about $20 \%$ is visible.
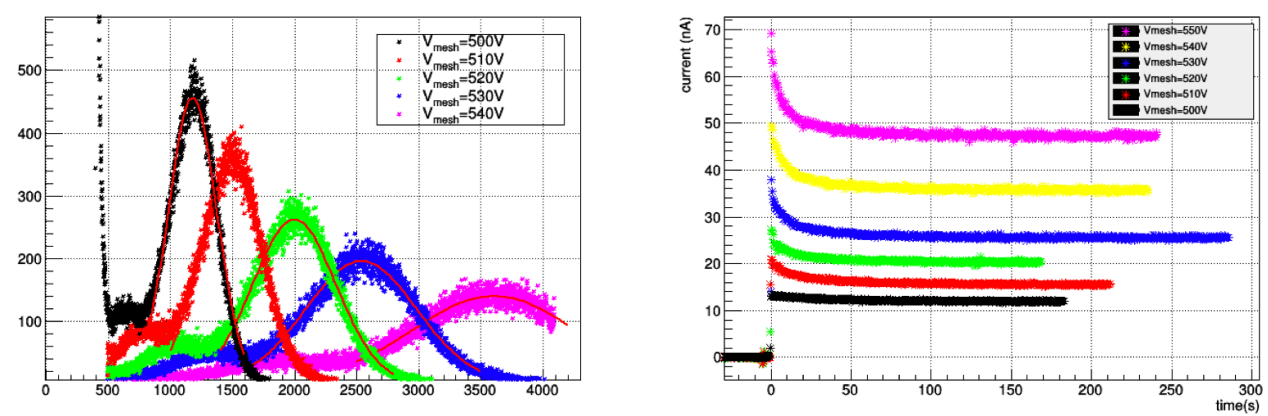

Figure 3: Left: Energy spectra for several amplification voltages. Right: Time evolution of the detector current.

Figure 4 left shows the relative electron transparecy, measured as relative value of the ${ }^{55} \mathrm{Fe}$ spectra peak position, as function of the fields ratio $\mathrm{V}_{\text {mesh }} / \mathrm{V}$ )drift. As expected the transparency reaches a plateau value above $95 \%$ for field ratio $>100$. The detector gain was measured with ${ }^{55} \mathrm{Fe}$ 
sources of different intensities and in different periods. The result is shown in fig. 4 right. At $\mathrm{V}_{\mathrm{amp}}=530 \mathrm{~V}$ the gain is above $5 \times 10^{3}$. We measured a $\sim 20 \%$ gain difference between the lowand high-intensity sources, giving a measured rate of 1.3 and $128 \mathrm{kHz}$, respectively. No significnt difference in gain was observed between measurment taken with the same (low-intensity) source after a 6 months interval.
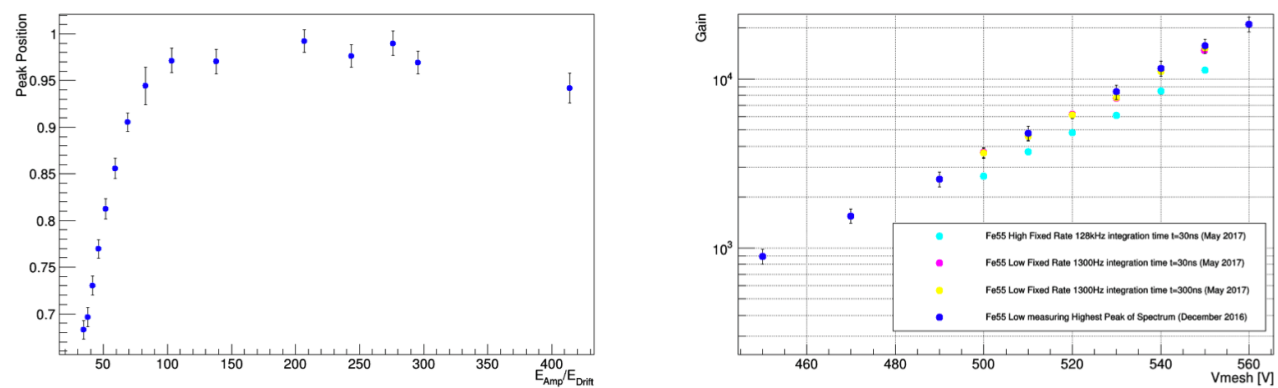

Figure 4: Left: Relative electron transparency as function of the field ratio. Right: Detector gain as function of the applied voltage to the mesh.

\subsection{Test with X-rays}

The detector was exposed to $8 \mathrm{keV}$ peak energy X-rays from a $\mathrm{Cu}$ target with collimators of 1,3 and $10 \mathrm{~mm}$ diameter. The intensity of the X-ray generator can be tuned by varyibg the excitation current, thus allowing to study the Micromegas response to different particle rates. For these measurments the detecor was operated at $\mathrm{V}_{\text {drift }}=2000 \mathrm{~V}$ and $\mathrm{V}_{\mathrm{amp}}=530 \mathrm{~V}$, if not otherwise stated.
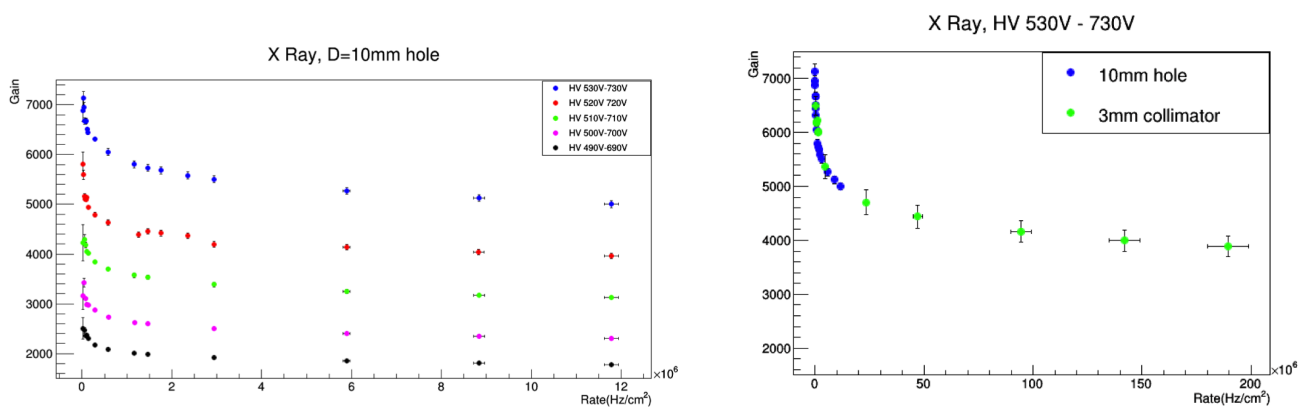

Figure 5: Detector gain as function of the particle rate.

Figure 5 left shows the amplification gain as function of the particle rate for five different values of the amplification voltage, measured using the largest collimator. All curves show maximum gain reduction of $\sim 20 \%$ at $12 \mathrm{MHz} / \mathrm{cm}^{2}$. At higher rates the gain goes further down, with smaller derivative, as shown in Fig. 5 right. The amplification gain of the detector was, hovewer, above 4000 at a rate of $150 \mathrm{MHz} / \mathrm{cm}^{2}$ with $\mathrm{V}_{\text {mesh }}=530 \mathrm{~V}$.

The variation of the gain across the small $(1 \mathrm{~mm})$ and large $(3 \mathrm{~mm})$ dimension of the pads was measured by moving the relative position between the detector and the $1 \mathrm{~mm}$ collimator of the $\mathrm{X}$-ray source along the $x$ and $y$ axis, respectively. The position scan was performed with a step of 
$0.1 \mathrm{~mm}$. Figure 6 shows the results for the $x$ and $y$ scans, respectively. A smooth modulation of the gain as function of the position along the pad is clearly visible, with a maximum variation of the gain, within a single pad, of about $4-5 \%$.
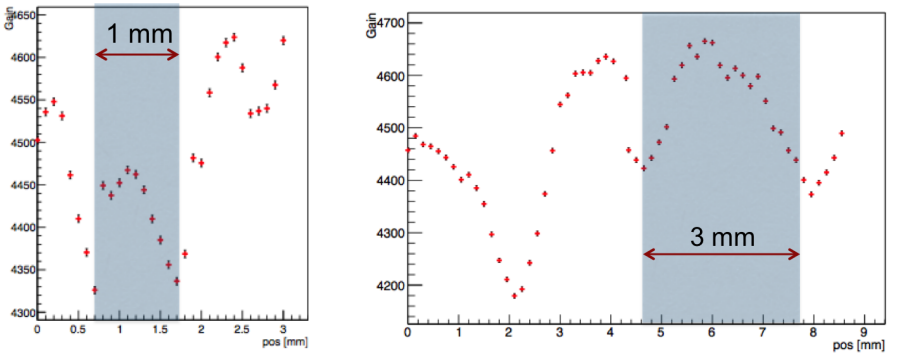

Figure 6: Detector gain at different positions on the readout pads across the $x$ (left) and the $y$ (right) directions.

\subsection{Test at particle beam}

The small-pad Micromegas was tested at the $\mathrm{H} 4$ experimental area at CERN with $120 \mathrm{GeV}$ pion and muon beams. The esperimental setup (fig. 7 left) included three additional standard bulk-Micromegas with $250 \mu \mathrm{m}$ pitch strips in $x$ and $y$ view for 2D tracking and a scintillator-based trigger system. All the detecttors were read out with front end card equipped with APV25 [6] hybrids. Data were aquired with the Scalable Readout System [7] interfaced with the acquisition PC by mean of a custom DAQ system (mmDAQ) developed at CERN.
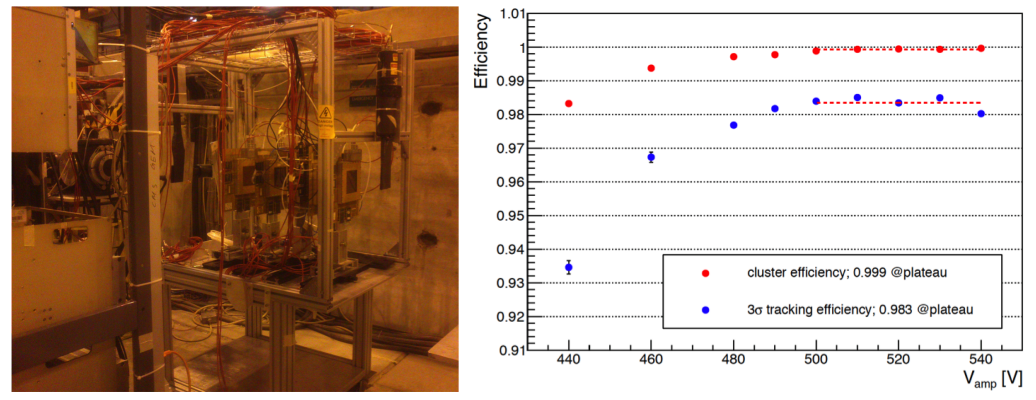

Figure 7: Left: Experimental setup for the test with particle beams at the SPS H4 beam line. Right: Detector efficiency as function of the amplification mesh (see text for the efficiency definition)

Events with single reconstructed clusters on all three tracking detectors have been used to measure efficiency and space resolution of our Micromegas. For each event a track is built out of the three cluster positions and extrapolated to the position of the small-pad Micromegas. Only events extrapolated to the active area of the detector are retained. Two types of efficiency have been defined: a) cluster efficiency: an event is classified as efficient if a good reconstructed cluster is found; b) $3 \sigma$ efficiency: an event is classified as efficient if a good reconstructed cluster is found within an acceptance window of $3 \sigma_{x}$ with respect to the position of the extrapolated track (see below for the definiton of $\sigma_{x}$ ). Figure 8 right shows the detector efficiency as function of the amplification voltage. Plateau values of $99.9 \%$ and $98.3 \%$ were measured for the cluster and $3 \sigma$ efficiency, respectively. 

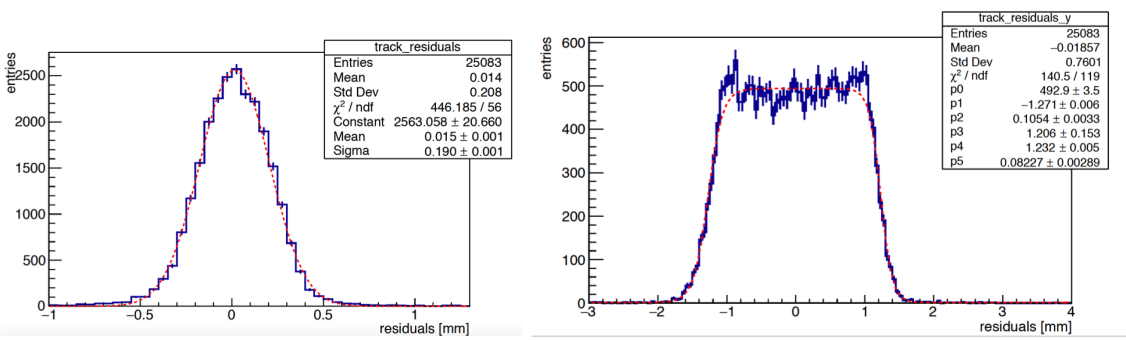

Figure 8: Residual distributions in $x$ (left) and $y$ (right).

The spatial resolution was obtained by measureing the residual, independently in $x$ and $y$, between the position of the extrapolated track and the reconstructed cluster on the detector, for cluster-efficient events, after an alignment procedure. When more than one cluster for the same event was found, the one with the smallest residual in $x$ was considered. Figure 8 shows the residual distributions in $x$ and $y$, respectively. The resolution in $x$ is extracted with a gaussian fit to the residual distribution, resulting in a measured value of $\sigma_{x}=190 \mu \mathrm{m}^{1}$. The residual distribution in $y$ has a box-like shape as a result of the pad pitch in $y(3 \mathrm{~mm})$ being much larger than the discharge footprint and the resolution of the tracking system. The full width at half maximum of the distribution (measured with a two Fermi-Dirac functions fit to describe the rising and falling edges) is found to be $1.5 \mathrm{~mm}$.

Finally, the dependence of the cluster size (the number of pads included in a cluster) was studied as a function of the expected particle position on the detector, as extrapolated from the tracking system. The result is shown in fig. 9: particles passing in the center of the pad have higher probability to fire an odd (one or three) number of pads (fig. 9 left), while particles passing close to the edge of the pad have higher chance to fire two pads (fig. 9 right). The probability differece is measured in about $20 \%$.
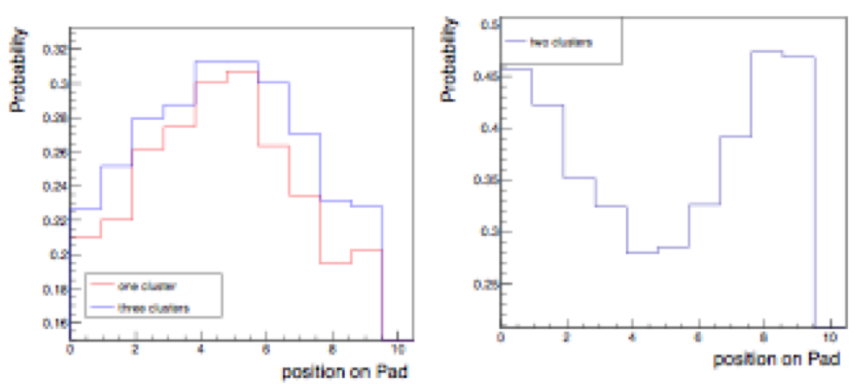

Figure 9: Left: Probability of cluster size equal to 1 (red) or 3 (blue) as function of the hit position within a pad in $x$. Right: Probability of cluster size equal to 2 as function of the hit position within a pad in $x$.

\section{Conclusions and future developments}

A resistive Micromegas with pads readout $\left(1 \times 3 \mathrm{~mm}^{2}\right)$ has been built and characterized at CERN. The measured efficiency is higher than $98 \%$ and the spatial resolution of the precise coor-

\footnotetext{
${ }^{1}$ This value includes the resolution of the tracking system estimated to be $\sim 40 \mu \mathrm{m}$
} 
dinate equal to $190 \mu \mathrm{m}$. The detecor showed a promising behaviour at very high rate, maintaining a gain of $\sim 4 \times 10^{3}$ at $150 \mathrm{MHz} / \mathrm{cm}^{2}$.

Further developments are focussed in two directions:

- optimisation of the resistive protection: a new prototype is now being tested with the same readout layout, exploiting a resistive layer made of diamond-like carbon (DLC) produced by sputtering technique and with a reduced number of resistive pads to readout pads connections;

- A new prototype is under construction, with APV25 electronics directly embedded on the backplane of the Micromegas board. This thecnique would simplify the routing from the readout pads to the conectors, offering a more compact and cheaper solution for the construction of small-pad Micromegas with a much larger active area and minimizing the dead space at the sides of the Micromegas board.

The two developments will be eventually merged into a DLC small-pad Micromegas detector with embedded electronics.

\section{Acknowledgements}

Authors are grateful to CERN GDD/RD51 laboratory, in particular to E. Oliveri, for continuous help and support. Special thanks go to the CERN PCB workshop, in particular R. De Oliveira and A. Teixeira.

\section{References}

[1] Nucl.Instrum.Meth. A640 (2011) 110

[2] CERN LHCC (2013) 006

[3] A similar plot was shown at the 'Workshop on requirements for future detector technologies in view of FCC-hh': https://indico.cern.ch/event/358198

[4] JINST 12 (2017) C03077

[5] Nucl.Instrum.Meth. A560 (2006) 405

[6] Nucl.Instrum.Meth. A466 (2001) 359

[7] JINST 8 (2013) C03015 